\title{
Application of Variance Components Modelling Approach to Tanzania Demographic and Health Survey Data; Identifying Determinants of Modern Contraceptive Uptake
}

Oliva Safari ( $D$ olivasafari@gmail.com )

Medical Teams International Tanzania, Department of Monitoring, Learning and Evaluation, Box 1 Kibondo Tanzania

\section{Dunstan R. Bishanga}

School of Public Health and Social Sciences, Muhimbili University of Health and Allied Sciences, P O Box 65001, Dar es Salaam

\section{Isambi Sailon}

IMS-NEI Global Secretariat, Gasabo Kacyiru - Kamatamu, KG590 ST, Kigali, Rwanda

\section{Research Article}

Keywords: Modern contraceptive use, TDHS, random component, random coefficient, nested structure

Posted Date: May 13th, 2021

DOl: https://doi.org/10.21203/rs.3.rs-484018/v1

License: (c) (1) This work is licensed under a Creative Commons Attribution 4.0 International License.

Read Full License 
Application of variance components modelling approach to Tanzania demographic and health survey data; Identifying determinants of Modern

\section{Contraceptive uptake}

\section{Oliva Safari $^{1}$, Dunstan R. Bishanga ${ }^{2}$ and Isambi Sailon Mbalawata ${ }^{3}$}

1. Medical Teams International Tanzania, Department of Monitoring, Learning and Evaluation, Box 1 Kibondo Tanzania

2. School of Public Health and Social Sciences, Muhimbili University of Health and Allied Sciences, P O Box 65001, Dar es Salaam, Tanzania; dbishanga@gmail.com

3. AIMS-NEI Global Secretariat, Gasabo Kacyiru - Kamatamu, KG590 ST, Kigali, Rwanda

Key Words: Modern contraceptive use, TDHS, random component, random coefficient, nested structure. 


\begin{abstract}
Background: Over time, Demographic and Health Survey (DHS) data remain valuable to examine variables relating to nationally representative population for low- and middle-income countries. In Tanzania, there are very limited DHS-based studies on the uptake of Modern Contraceptive Use (MCU). Present studies were focused on individuals' levels measurements, yet research have shown the MCU variations still exists to other levels of populations. In this study, we aimed to use the variance component modelling approach to explore variation on MCU at PSU and region levels while considering survey sample weighting.
\end{abstract}

Methods: Using DHS 2016-2017 in Tanzania, we study various variance structure and the respective variation on MCU to more than 5174 Women of Reproductive Age (WRA) 15-49 years. Firstly, a single variance component was used, followed by its extension to random coefficient model and we tracked changes in the models.

Results: There was an influence of random variations on MCU on the levels of populations much explained by PSU-level clustering than region. On the fixed part, age of a woman, education level, husband education level, desire to have children, exposure to media and wealth index were the important determinant for MCU among WRA in Tanzania. For example, the odds of MCU among middle ages women (20-29 and 30-39) were $29 \%$ and $90 \%$. Also, odds of MCU increases with an increase to media exposure and primary and secondary school women had higher chances for MCU. We also found assessing effects of covariates at two different nested levels eventually resulted in different estimates.

Conclusion: This study highlighted on utility of accounting for variance structures in addressing various sources of variations on MCU while using DHS national level data. Apart from MCU, the TDHS data has been widely applied to examined other variables pertaining to public health issue. Thus, this approach could be considered a better modelling technique for the DHS studies. 


\section{Background}

Many thanks to literatures studied on MCU in middle-and-low-income countries, particularly in the Sub-Saharan Africa and Tanzania. To increase the uptake of MCU remains crucial in addressing maternal and child health services despite switching from Millennium to Sustainable Developments Goals [1-3]. The significant importance of MCU over natural methods is primarily seen in preventing unplanned pregnancies with global reductions from $43 \%$ in 1995 to $40 \%$ in 2012 . This was influenced by increased demand for family planning, as demonstrated by the increase in demand for MCU from $68 \%$ to $78 \%$. MCU help in control of fertility rates as discussed in [4] because of the practice of child spacing. A recent global report shows that MCU has increased from 54\% to 57\% from 1990 to 2015 [2]. In Sub-Saharan African (SSA) usage remained persistently low; around 23\% and 24\% respectively. The same report showed a higher usage rate in developed countries, with $80 \%$ and above in the UK and China, and 75\% in North America. Again, in this report it is pointed out that in developed countries maternal and child health status are improved[5,6].

So, what is the problem? In Tanzania, 24\% of women of reproductive age are facing a crucial unmet need for family planning. This is twice the world average (12\%) and stems from a low (32\%) use of contraceptives, particularly modern contraceptives [7]. However, according to this report $50 \%$ of maternal mortalities within the country is attributed to low and ineffective uptake of MCU. In addition, Tanzania is among the leading African countries in terms of fertility rations ( 4 children per woman). Both maternal mortality and fertility rates could be controlled by effective MCU $[4,8]$. In recognizing the need for MCU, the Government of Tanzania imposed many strategies to double the number of contraceptive users by the year 2015. This included providing high-quality, accessible, acceptable, and affordable Family Planning (FP) services for young people, strengthening the supply chain for FP commodities, increasing male involvement in FP issues and a mass media campaign [7]. The uptake of 
MCU remains extremely low in Tanzania and there is a marked regional variation from $6.8 \%$ to $50.8 \%$ in Kusini Pemba and Ruvuma respectively [7, 9].

Using DHS data, studies have reported on various covariates associated with MCU, including education levels among women of reproductive health, age levels, exposure to media and wealth levels as discussed by Ferede and Ejembi $[10,11]$. In Tanzania, a TDHS-based study identified factors associated with MCU including difference in age levels among partners, partners levels of educations and women empowerments [12]. However, other studies among different populations in Tanzania reported similar characteristics associated with MCU uptake [13-16]. Most of these studies focuses on a small-scale sample with limited targeted populations (secondary school students, university population and HIV groups), that are less representative of overall WRA with in the country but only to specific group that study was conducted in respective research area. Furthermore, when the TDHS data is used as the representative of WRA then modelling approaches deployed unweighted sampling modelling methods. Despite a study reported that survey data weighting has slight inferential difference with unweighted data[17], It is still important that the modelling of DHS(Demographic and Health Survey) should consider not only sample weighting, but also should account for the hierarchical nature of the data resulted from multistage sampling. Also, MCU uptake may vary from point to point in respect to individuals' characteristics, thus it is important for public health research to consider statistical approaches indicating evidence-based variations. In this article we aimed to use survey sample weighting and variance components modelling approaches, in addressing valuable variations resulting from hierarchical levels of populations in respect to demographic, socio-demographic and other related characteristics. Specifically, we aimed to

I. estimate size of both the PSUs and regional heterogeneity on MCU.

II. identify determinant of MCU while accounting for the variance structures. 
III. determine the effect of varying covariate model on MCU.

IV. draw important lessons for public health policy, applications, and practises.

\section{Methods}

\section{Study settings}

This study used data from the Tanzania Demographic and Health Survey (TDHS) for 2016/2017 survey as obtained from DHS website: www.dhsprogram.com. The TDHS surveys are normally conducted every five years and to nationally represent health related variables of the populations under study. The sample selections were based on two stage sampling with enumeration areas (EAs) which were streets in urban or villages in rural as the primary sampling unit i.e., the PSUs, followed by selection of households which contains women of reproductive age. The survey was assigned a sample weight variable (HV005) to account for risk of over sampling or under sampling while strata are presented by variable (V023); more details on variables can be obtained in DHS manual [18].

A total of 13266 women of reproductive age (WRA) were sampled. A total of 5077 were dropped as they are not married; 1862 women who desired children in next two years were omitted, 188 infecund women were dropped, and 965 women who were pregnant were also removed from study as they are not recommended for MCU uptake. Of all surveyed, we remained with 5263(weighted sample) women eligible for MCU study.

\section{Variables}

The TDHS program usually collect a lot of information including the status of MCU by any modern method. Dependent variable was obtained by dichotomization process with 1 for women used one of the following; injectable, pills, sterilizations, Inter- Uterine Device (UID), condoms and lactation amenorrhea and 0 otherwise[7]. Covariates used includes woman's age(15-19,20-29,30-39,40-49), woman's educational level(never to schools, primary secondary+), exposure to media for radio, television, newspaper(not exposed at all, exposed to 
at least one, exposed to at least two, exposed to all), wealth index(poor, middle, rich), urbanrural place of resident, parity(no child, 1child, 2children, children $3+$ ) [7, 10, 11, 19, 20].

\section{Statistical considerations}

\section{The Variance Component modelling approach to $\mathrm{MCU}$}

The variance components were used in 1918 by Fisher in genetics studies and later in 1931 by Tippest in sampling, to address best methods of dealing with, between and within group observation. The approach has increased in popularity in a variety of fields such as agriculture; biology for laboratory trials and medicine; education, and engineering, experimental and survey or panel data to address many sources contributing to variations of a characteristic or a variables or process $[21,22]$. However, approach is commonly used in connection with clustering, nested models, mixed effects models, multilevel models, and hierarchical or random effect models [23, 24].

Using Leyland and Goldstein[22], let's consider a grouping or hierarchical data structure from TDHS in which individuals are sampled from 608 PSUs the Primary Sampling Unit (PSUs) across the whole country, say 608 group of PSUs representing a whole population of WRA in the country. That is, individual $\mathrm{i}\left(\mathrm{i}=1,2,3 \ldots \ldots, i_{k}\right), i_{k}=5174$ (the total sample size) sampled from PSU (villages and streets) $\mathrm{j}\left(\mathrm{j}=1,2,3 \ldots \ldots, j_{q}\right), j_{q}=608$. Our interest might be on population forming a sample of PSUs than PSUs themselves, thus PSUs are considered as random sample from infinite hypothetical population of PSUs. Infinite hypothetical in a sense that, the PSUs' population is unknown unlike population defining individuals. To model such data, we need to consider two kind of variations: that between individuals in the same PSU and between PSUs themselves, giving the following model expression:

$Y i j=\beta_{0 j}+u_{0 j}+e_{i j}$ 
where, $Y_{i j}$ is $i^{\text {th }}$ individual observation on use of modern contraceptive in the $j^{\text {th }} \mathrm{EA}, \beta_{0 j}$ given as $\beta o+u o j$ defined $\beta_{0 j}$ as random parameter of a population of quantities with mean value given as $\beta o$. The $\beta o$ describe a fixed part of the model. It is also called a random intercept which is the average value of $Y_{i j}$ when there are no covariates in a model or the mean value of MCU across PSUs with zero influence of predictors. $u_{0 j}$ is the random component applying to all individuals in $j^{\text {th }}$ PSU with mean value of 0 and variance $\delta_{u 0}^{2} \cdot e_{i j}$ is another random component for $i^{\text {th }}$ woman in the $j^{\text {th }} \mathrm{PSU}$; its mean is 0 and variance $\delta_{e}^{2} \cdot u_{0 j}$ and $e_{i j}$ are random parameters with correlations 0 between themselves, forming a random part of the model and partitioning the variance into two parts $e_{i j}$ and $u_{0 j}$. Now, the fact that variance is divided into multiple levels of $\delta^{2}$ s leads to the term variance components (two level variances). The two levels variances come from variances associated with the individuals and the PSUs respectively, allowing for variation on MCU across two levels. The two terms $\left(u_{0 j}\right.$ and $e_{i j}$ ) corresponding respective expectation's, which is also equivalent to mean is given as;

$E\left(e_{i j}\right)=E\left(u_{0 j}\right)=0$

In this study we model MCU, this is binary composite variable coded as 1 and 0 for non-user

say $Y_{i j} \begin{cases}1 & \text { if } Y_{i j}=M C U \\ 0 & \text { otherwise }\end{cases}$

Thus, expectation or conditional probability that there is MCU given value of single predictor variable $X_{1 i j}$, is defined as:

$E\left(Y_{i j}, X_{1 i j}\right)=\beta_{0}+\beta_{1} X_{1 i j}$

Since (2) will be zero. This can be regarded as probability of success when $Y_{i j}=1$ given covariate $X_{1 i j}$ 
$P\left(Y_{i j}=1 / X_{1 i j}\right)=P\left(\beta_{0}+\beta_{1} X_{1 i j}+u_{0 j}+e_{i j}\right)=1, X_{1 i j}=P\left(e_{i j}-\beta_{0}-\beta_{1} X_{1 i j}-u_{0 j}\right)$

and equals to;

$$
F\left(\beta_{0}+\beta_{1} \mathrm{X}_{1 \mathrm{ij}}+\mathrm{u}_{0 \mathrm{j}}\right)=\pi_{\mathrm{ij}} \quad(\text { the success probability-when } \mathrm{MCU}=1)
$$

Equation (6) is defined as $\pi_{i j}$ which indicate the success probability function i.e. when there is MCU given exposure variable. In conclusion, $\pi_{i j}$ and $1-\pi_{i j}$ define success and failures values for binomial probability since there are two possibilities for MCU uptake; use (when there is MCU uptake) and non-use (when there is no uptake) respectively. From (4), we have binary response variable which follow under logistic link function providing following models expression;

$\log \left(\frac{\pi_{i j}}{1-\pi_{i j}}\right)=\beta_{0}+\beta_{1} X_{1 i j}+u_{0 j}$

or else, $\quad \pi_{i j}=\frac{\exp \left(\beta_{0}+\beta_{1} X_{1 i j}+u_{0 j}\right)}{1+\exp \left(\beta 0+\beta_{1} X_{1 i j}+u_{0 j}\right)}$

In another scenario, we might also be interested to see whether the relationship between MCU and exposure variable $X_{1}$, say wealth may not be fixed across groups. This extend a random intercept model to a random coefficient, by relaxing the assumption that influence of variable wealth to MCU is same across PSUs (villages and streets) and region levels. Thus, the coefficient of variable wealth $\left(\beta_{1}\right)$ which was initially a fixed effect, should be containing random parameter; $\beta_{1}$ is presented as $\beta_{1}+u_{01 j}$ and $u_{01 j}$, present a random quantity that allows effect of wealth to be varying across all women's PSUs of residence. But $u_{0 j}$ and $u_{01 j}$ are assumed to be correlated to estimate the covariance matrix for this correlation and $\delta_{\mathrm{u} 01}^{2}$ is the variance associated with random quantity $u_{01 j}$ for varying coefficient of wealth on the levels of PSUs. Now, consider that the effect of wealth is random across PSUs levels, this extends the simple random intercept model to random slope or coefficient model. In general, if we have $n$ covariates $\left(X_{1}, X_{2}, X_{3}, \ldots \ldots, X_{n}\right)$, and other $\left(X_{1}, X_{2}, X_{3}, \ldots, X_{p}\right), p$ covariates 
associated with varying slope across PSUs on MCU, in typical statistical model with logit-link function this can be expressed as;

$$
\log \left(\frac{\pi_{i j}}{1-\pi_{i j}}\right)=\beta_{0}+\beta_{z} X_{z i j}+\beta_{b j} X_{b i j}+u_{0 j}+\cdots+u_{0 b j}
$$

where $\beta_{\mathrm{bj}}=\beta_{\mathrm{b}}+\mathrm{u}_{0 \mathrm{bj}}$ and $b=1,2,3 \ldots \ldots p$ (number of variables considered as random coefficient, which is wealth for this analysis) and $z=1,2,3 \ldots \ldots n$ (fixed effect parameters associated with covariates which are equal to number of variables under study). Subscripts $n=6$ which are the selected predictors variables and $p=1$ which is for one variable regarded as random coefficient(wealth)

So far, in the TDHS the PSUs were clustered within regions or province creating a further nesting structure. Accordingly, the variance term is partitioned into three more levels, with $\mathrm{u}_{0 \mathrm{jk}}$ representing a random component associated with $i^{\text {th }}$ individual in $j^{\text {th }}$ PSU and $k^{\text {th }}$ region. So, the respective random parameters are $e_{i j}, u_{0 j}$ and $u_{0 k}$ which gives three levels of variance components model for individuals, PSUs (villages and streets) and regional with variances terms $\delta_{e}^{2}, \delta_{u 0}^{2}$ and $\delta_{u 1}^{2}$ respectively. The equational model is given by;

$$
\log \left(\frac{\pi_{i j k}}{1-\pi_{i j k}}\right)=\beta_{0}+\beta_{z} X_{z i j}+\beta_{b j} X_{b i j}+u_{0 j}+u_{0 b k}
$$

Figure 1 shows the nested data structure of TDHS with two levels (left) and three levels (right).
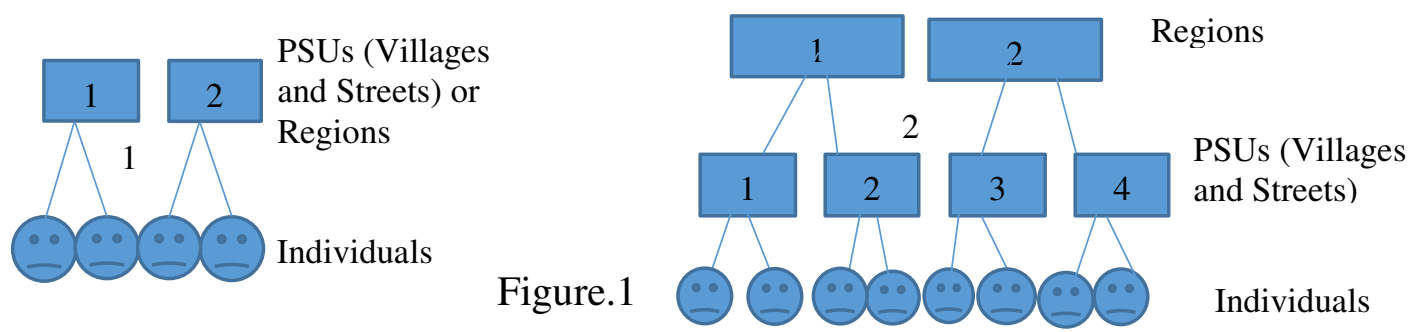

Figure.1, The TDHS data grouping structures two scenarios; (1). Individuals nested with in the villages and streets or individual nested with regions (two levels). (2); Individuals and villages and streets nested in regions (Three levels) 


\section{Analysis}

The TDHS data is structured or grouped observations thus, obviously we must expect many chances for characteristics of women in one group level say a region to relate with each other, rather than to the other regions [22].

We fitted three generalised linear models to data, by first start with a plain model with no covariates but contain random intercepts only. Model 1 include one cluster variable PSU, Model 2 contain another cluster variable region and Model 3 include both two cluster variables from Model 1 and Model 2. Thus Model 1 and Model 2 considered as two-level variance component models with two level variances. Model 3 had three level of variances components, thus term it as three level variance component model. Note that, the analysis of empty model helps in exploring proportions of variance attributed to clustering effects [25, 26] The best fit model was assessed using Bayesian deviance information criteria (DIC), Akaike information criteria (AIC) and loglikelihood value. Also, we used the 95\% confidences intervals (CIs) to assess the significant of the parameter that in the normal statistical practice, if $95 \%$ CIs contain point estimate, the p-values are more likely to show no associations. Finally, a model with random coefficient variable was presented to appreciate significant variability of the characteristic on MCU across groups.

\section{Results}

\section{Background characteristics}

Table 1 shows background characteristics of sample based on TDHS 2015/2016. Out of 5263 examined sample prevalence of MCU was almost 44\% (95\%CI; 0.425-0.452). Majority were at the age of 20-29 and 30-39 years. More than 70\% of women had primary education, while 
$31.36 \%$ were from urban areas. Likewise, $70 \%$ of women's husbands had only primary

educations, $19 \%$ secondary and $11 \%$ had never ever been to school.

Table 1, Baseline characteristics of unpregnant married women, infecund and with no desire to have children in next two years, TDHS 2015/2016

\begin{tabular}{|c|c|c|}
\hline Characteristics & $\mathrm{n}$ & $\%(95 \% \mathrm{CI})$ \\
\hline \multicolumn{3}{|l|}{ Modern contraceptive use } \\
\hline Not using & 2,954 & $56.13(54.8 ; 57.5)$ \\
\hline Use & 2,309 & $43.87(42.5 ; 45.2)$ \\
\hline \multicolumn{3}{|l|}{ Age in years groups } \\
\hline $15-19$ & 289 & $5.49(4.90 ; 6.10)$ \\
\hline $20-29$ & 1929 & $36.65(35.4 ; 38.0)$ \\
\hline $30-39$ & 1799 & $34.19(32.9 ; 35.5)$ \\
\hline $40-49$ & 1246 & $23.67(22.5 ; 24.8)$ \\
\hline \multicolumn{3}{|l|}{ Woman educational levels } \\
\hline 0. Never & 983 & $18.68(17.7 ; 19.8)$ \\
\hline 1. Primary & 3512 & $66.73(65.4 ; 68.0)$ \\
\hline 3. Secondary+ & 768 & $14.59(13.7 ; 15.6)$ \\
\hline \multicolumn{3}{|l|}{ Parity } \\
\hline None & 21 & $0.41(0.30 ; 0.60)$ \\
\hline One to two & 1768 & $33.59(32.3 ; 349)$ \\
\hline Three to four & 1717 & $32.63(31.4 ; 33.9)$ \\
\hline Five+ & 1756 & $33.37(32.1 ; 34.7)$ \\
\hline \multicolumn{3}{|l|}{ Place of resident } \\
\hline Urban & 1,650 & $31.36(30.1 ; 32.6)$ \\
\hline Rural & 3,613 & $68.64(67.4 ; 69.9)$ \\
\hline \multicolumn{3}{|l|}{ Husband desire for children } \\
\hline Don’t knows & 1,571 & $0.41(028.6 ; 0.311)$ \\
\hline Both want same & 2,046 & $33.59(37.6 ; 40.2)$ \\
\hline Husband desire more & 1,321 & $32.63(23.90 ; 26.30)$ \\
\hline Husband desire less & 325 & $33.37(5.60 ; 6.900)$ \\
\hline \multicolumn{3}{|l|}{ Age difference with man } \\
\hline Woman older than man & 212 & $4.03(3.50 ; 4.60)$ \\
\hline Same age level & 179 & $3.41(3.00 ; 3.90)$ \\
\hline Man old for 10- & 3822 & $72.66(71.4 ; 738)$ \\
\hline Man old for $10+$ & 1047 & $19.9(18.8 ; 21.0)$ \\
\hline \multicolumn{3}{|l|}{ Husband education level } \\
\hline No education & 576 & $10.94(10.1 ; 11.8)$ \\
\hline Primary education & 3707 & $70.44(69.2 ; 71.7)$ \\
\hline Secondary and higher & 980 & $18.62(17.6 ; 19.7)$ \\
\hline \multicolumn{3}{|l|}{ Wealth index } \\
\hline Poor & $2,000.71$ & $38.02(36.7 ; 39.3)$ \\
\hline middle & $1,030.89$ & $19.59(18.5 ; 20.7)$ \\
\hline Rich & $2,231.35$ & $42.4(41.1 ; 43.7)$ \\
\hline \multicolumn{3}{|l|}{ Exposure to media } \\
\hline Not exposed & 893 & $16.96(16.0 ; 18.0)$ \\
\hline Exposed to at least one & 1567 & $29.78(28.6 ; 31.0)$ \\
\hline Exposed to at least two & 1504 & $28.57(27.4 ; 29.8)$ \\
\hline Exposed to all & 1299 & $24.68(23.5 ; 25.9)$ \\
\hline
\end{tabular}




\section{PSU and Regional variation on MCU}

Table 2 shows results from three random component models with only random intercepts and dependent variable MCU. Based on all AIC, BIC and log-likelihood values, Model 3 with both PSU and region as clustering variables was best fitted the data compared to Model 1 and Model 2. The AIC and BIC for Model 3 were 6793.758 and 6813.413 smaller than Model 1 and Model 2. Also, Model 3 had a bigger log-likelihood value compared to all models which implies there was both PSU and regional variabilities on MCU. Similarly, the Model 1 had a better fit than Model 2 that means there was substantial PSU level variation on MCU than regional level variation.

In Model 3, it was found that PSU level variance $\left(\delta_{u 0}^{2}=0.466\right)$ was bigger than regional $\left(\delta_{u 1}^{2}=0.387\right)$ level variance, this implies that there was significant PSU level variability than region level variability on MCU. The intra-class correlation coefficients (ICCs) which the measure for relatedness of individuals characteristics within a same group was 0.205 for PSU and 0.094 for region. That mean, about $21 \%$ and $9 \%$ variations on MCU among WRA in Tanzania were attributable to clustering from PSU and region, respectively.

Table 2, Two and three levels empty random components models for MCU

\begin{tabular}{llll}
\hline Parameter & Model 1(PSU) & Model 2(Region) & Model 3(PSU and region) \\
\hline$\delta_{u 0}^{2}\left(\right.$ Variance $\left.u_{0 j}\right)$ & $0.74(0.308 ; 0.566)$ & & $0.466(0.308 ; 0.566)$ \\
$\delta_{u 1}^{2}\left(\right.$ Variance $\left.u_{0 k}\right)$ & & $0.356(0.22 ; 5644)$ & $0.387(0.267 ; 0.849)$ \\
ICC (region) & & $0.098(0.0634 ; 0.1464)$ & $0.094(0.0579 ; 147)$ \\
ICC (villages) & $0.18(0.140-0.224)$ & & $0.205(0.160 ; 0.261)$ \\
AIC & 6885.68 & 6961.31 & 6793.758 \\
BIC & 6898.78 & 6974.413 & 6813.413 \\
Log-likelihood & -3440.84 & -3478.655 & -3275.67 \\
\hline
\end{tabular}




\section{Determinants of modern contraceptive use accounting for variances structure.}

Age of a woman, education level, husband's education level, desire to have more children, exposure to media and wealth index level were significantly associated with MCU. The odds of MCU were almost 2-times higher among women with age 20-29 and 30-39 years compared to the 15-19 years. The odds of MCU for women who had primary and secondary education were 1.22 and 1.08 compared to never been to school. This means women who had primary education were $22 \%$ and $8 \%$ more likely to use modern contraceptives than women who never ever been to school, respectively. Exposure to at least two media source had positive association on MCU, that women with exposure to at least radio or television or newspapers, and women with exposure to other two or three medias were $105 \%, 109 \%$ and $154 \%$ more likely to use modern contraceptive than women with no exposure to media. Similarly, the middle and rich women were $107 \%$ and $154 \%$ more likely to use modern contraceptive while women with husbands sired to have more children were 68\%less likely to use modern contraceptive (Table 3).

Table 3, Variance components model with a random intercepts and random coefficient examining the determinants of MCU, TDHS, 2015/2016

\begin{tabular}{|c|c|c|}
\hline & $\begin{array}{l}\text { Random intercept Model } \\
3\end{array}$ & Random coefficient Model 3 \\
\hline Fixed part & $(95 \% \mathrm{CI})$ & $(95 \% \mathrm{CI})$ \\
\hline \multicolumn{3}{|l|}{ Age in years groups } \\
\hline \multicolumn{3}{|l|}{$15-19$} \\
\hline $20-29$ & $1.94(1.244 ; 3.024)$ & $1.95(1.261 ; 3.009)$ \\
\hline 30-39 & $2.28(1.372 ; 3.803)$ & $2.29(1.379 ; 3.809)$ \\
\hline $40-49$ & $1.41(0.819 ; 2.432)$ & $1.40(0.810 ; 2.409)$ \\
\hline \multicolumn{3}{|c|}{ Woman educational levels } \\
\hline \multicolumn{3}{|l|}{0 . Never } \\
\hline 1. Primary & $1.22(0.985 ; 1.507)$ & $1.20(0.963 ; 1.488)$ \\
\hline 3. Secondary+ & $1.08(0.776 ; 1.497)$ & $1.06(0.765 ; 1.477)$ \\
\hline \multicolumn{3}{|l|}{ Parity } \\
\hline None & & \\
\hline One to two & $1.39(0.471 ; 4.111)$ & $1.39(0.464 ; 4.140)$ \\
\hline Three to four & $1.45(0.484 ; 4.335)$ & $1.45(0.478 ; 4.383)$ \\
\hline Five+ & $1.40(0.443 ; 4.403)$ & $1.39(0.436 ; 4.454)$ \\
\hline \multicolumn{3}{|l|}{ Place of resident } \\
\hline Urban & & \\
\hline Rural & $1.10(0.848 ; 1.429)$ & $1.12(0.863 ; 1.442)$ \\
\hline
\end{tabular}


Husband desire for children

Don't knows

Both want same $\quad 0.82(0.691 ; 0.972)$

$0.82(0.686 ; 0.972)$

Husband desire more

$0.64(0.489 ; 0.850)$

$0.65(0.493 ; 0.865)$

Husband desire less

$0.84(0.644 ; 1.097)$

$0.83(0.630 ; 1.098)$

Age difference with man

Woman older than man

Same age level

$1.16(0.779 ; 1.717)$

$1.17(0.780 ; 1.763)$

Man old for 10-

$0.83(0.646 ; 1.065)$

$0.83(0.642 ; 1.070)$

Man old for $10+$

$0.73(0.536 ; 1.003)$

$0.73(0.535 ; 1.006)$

Husband education level

No education

Primary education

Secondary and higher

$1.58(1.232 ; 2.019)$

$1.52(1.174 ; 1.980)$

Wealth index

$1.36(0.929 ; 1.981)$

$1.34(0.921 ; 1.939)$

Poor

middle

$1.57(1.260 ; 1.949)$

$1.60(1.284 ; 1.987)$

Rich

$2.14(1.617 ; 2.826)$

$2.18(1.632 ; 2.923)$

Exposure to media

Not exposed

Exposed to at least one

$1.05(0.763 ; 1.451)$

$1.05(0.759 ; 1.445)$

Exposed to at least two

$1.39(1.091 ; 1.770)$

$1.38(1.087 ; 1.747)$

Exposed to all

$1.54(1.189 ; 2.002)$

$1.54(1.187 ; 1.988)$

Random part

\begin{tabular}{lll}
\hline$\delta_{u 0}^{2}=\operatorname{Variance}\left(u_{0 j}\right)$ & $0.360(0.193 ; 0.671)$ & $0.718(0.413 ; 1.248)$ \\
$\delta_{u 1}^{2}=\operatorname{Variance}\left(u_{0 k}\right)$ & $0.341(0.215 ; 0.541)$ & $0.381(0.191 ; 0.762)$ \\
$\delta_{\mathrm{u} 01}^{2}=\operatorname{Var}-\operatorname{Cov}($ & & $0.137(0.049 ; 0.377)$ \\
$\left.\mathrm{u}_{01}, \mathrm{u}_{0 \mathrm{j}}\right)$ & \\
$\delta_{u 11}^{2}=\operatorname{Var}-\operatorname{Cov}($ & & $0.022(0.002 ; 0.206)$ \\
$\left.\mathrm{u}_{11}, \mathrm{u}_{0 \mathrm{k}}\right)$ & $0.090(0.050 ; 0.156)$ & $0.087(0.045 ; 0.161)$ \\
ICC (region) & $0.176(0.127 ; 0.237)$ & $0.251(0.179 ; 0.338)$ \\
ICC (villages) & 6590.534 & 6577.618 \\
AIC & 6754.309 & 6761.046 \\
BIC & -3270.2668 & -3260.809 \\
Log-likelihood & &
\end{tabular}

\section{The effect of varying covariate model on MCU}

It is possible for the fixed effect characteristics on MCU to vary randomly across PSU and regional levels to consider a model as varying covariate model. To illustrate this, a random coefficient Model 3 was fitted to data and allow for the possibility that the influence of wealth on MCU not be fixed as it was fitted before (Table 3).

The loglikelihood values for Model 3 with the random coefficient and Model 3 with random intercepts were -3260.809 and -3270.2668 respectively, indicating significant important to 
retain a random coefficient variable to the model. That is, Model 3 with random coefficient best fitted the data than Model 3 without a random coefficient variable. The variance covariance between wealth index and region was 0.022 , implying there was a positive heterogeneity for wealth index on MCU across regional levels by $2 \%$. Likewise, the variance covariance for wealth index and PSU was 0.137 , that means there was nearly $14 \%$ positive significant variation of wealth across PSU level $\left(\delta_{\mathrm{u} 10}^{2}=0.137 ; 95 \% \mathrm{CI}\right.$ : 0.49-0.377). However, adding a random coefficient component for wealth index result to confounding effects to other variables in the model. For example, woman educational level was significantly associated with MCU where women with primary school education $22 \%$ chance for MCU had, but after accounting for random coefficient variable, there was insignificant association. Also, there were significant increase in the ICCs values for PSU from $176 \%$ to $254 \%$ and reduction for regional from $9 \%$ to $8 \%$ when comparing two respective models. In general, the random coefficient model, has results to confounding effects on the entire Model 3 parameters. But, age of woman, education level of man, age differences between a woman and a man, desire to have more children, exposure to media and wealth index had remained the significant covariates for MCU among WRA in Tanzania.

\section{Strengths and Limitations}

Given the nature of cross-sectional study design; similarly, for the TDHS used in this study, it was challenging in making casual inferences due to difficulty in determining the sequence of occurrence between set of selected exposure variables such as age, woman educational level, and outcome of interest (MCU).

This study was not able to capture characteristics relating to knowledge, attitude, and practices on MCU in Tanzania. Also, it was not possible to report on neighborhood or community-level characteristics on MCU with this study. 
The main strength for TDHS data is based on representation of entire population of the WRA in the country and contained nested data structure. With the nesting data structure of TDHS, this study was able to consider respective associated variance structures in relation to determinants of MCU uptake.

\section{Discussion}

Our aim with this article is to make use of a variance components modelling approach to exploit the structure of the TDHS data in addressing MCU. We explored various techniques including analysis of simple models to extensions to varying covariate model and obtained a best fit overall model. In general, we found presence of both fixed and random effects influencing MCU. With fixed effects, age of a woman, education's level, man's education levels, exposure to media, wealth index and desire to have more children are the important covariates statistically significantly associated with MCU. We also found presence random variations in forms of random intercepts and random coefficient.

During analysis of random intercept model, an empty random component Model 3 showed evidence for variations on MCU at both groups' levels. The significant of random intercepts compared to traditional models was also reported by many authors $[10,11,27-29]$. In the analysis of random coefficient model, it was noted that more variations on MCU were attributed to PSU clustering than region. The reason might be due to existence in variations in individual characteristics at PSU level than region. For example, characteristics like wealth and education levels may vary between villages and streets that some of these PSUs contained more educated individuals as well as more wealthy population than others. The differences in characteristics may have impact on MCU uptake. Studies from Nigeria, Zimbabwe and Ethiopia have reported positive influence of community levels on MCU estimated at PSU level [10, 11, 30]. However, most of these studies were conducted with only one cluster variable (PSU), which limits for their 
results to be used in the setting where there are two levels of grouping variables (PSU and region) used as for the analysis of this study.

Also, much variation from PSU than region clustering extended to the analysis of varying covariate model (Model 3 with rando coefficient), perhaps this could reflect an existence of effect of neighbourhood or grouping effect more due PSU clustering than the region, that is there is more close relationships of individual's characteristic at PSU level than region. Studies have suggested an evidence for presence of effects of neighbourhood or community on health related outcomes that is measured at PSUs levels(the low level cluster) in setting of this study [31-33]. On the other hand, the ICCs for PSU was larger than ICC for region, perhaps this was because there was smaller number of individuals at region than PSU clusters as the ICCs were ever re reported to be inversely related with cluster size as presented in [9].

As far as for group effects on MCU, the effects of covariates are notably seen when fixed effects were introduced to an empty variance component model and when more random parameters are added to models. There were changes in all parameters in the models including the random intercepts and log-like hood values, that differ from models contained fixed effect with empty models. Ferede and Ejembi et al. shown changes in values of random intercepts as a result of adding covariates during model selection $[10,11]$. However, covariates such as age of a woman, education level of a woman, wealth index, and media exposure had remained to be important determinants of MCU [34-36] although later on women's education level was found to have no association. This could be that the association between woman education level and MCU was distorted by the others variables in the model, and similar effect reported by the study conducted by Ngome and Odemwegw [37]. Also, because these characteristics were significant in past studies $[7,10,11,19,20]$, similar significant was expected in this study. 


\section{Public Health policy and Research Practises Recommendations}

So, what is the lesson from addressing MCU? There exists considerable variation in MCU due to hierarchical structure of population at PSU and regional levels. To our best, we have tried to illustrate that it is possible for population characteristic such levels of wealth index to have random influence on MCU from one hierarchy of population to another. This emphasis on presence of dynamics in populations characteristics and the attributed effect to health outcomes such us MCU. For the public health policy, the implications would be, health policy makers should be aware of between group variations (PSUs and regions) in respect to variations in population's characteristics on MCU over time. This is because such variational impacts do exist not only on MCU but also to the other characteristics of public health importance in the country and using DHS data may be following under similar situation like MCU, given that DHSs have been used widely over time for government planning purposes.

Furthermore, after taking into considerations of nested data structure and associated random quantities we found difference estimates between models to the overall best fit model. For example, when two levels data fitted, results differed from when three level data are fitted to data. Also, the parameter estimates from model with random intercept differed from model with both random intercept and random slope model. This may be a key lesson to Statistician to be careful in the selection of appropriate modelling technics with respect to the data structure for efficient estimates in making inferences. However, because this is survey-based data, one may be interested to use survey modelling approach but since it gives zero presentation of any form of random effects parameters it would be difficult to infer on various variance structures associated with data. The robust modelling methods for cluster would be useful; however, this method treats cluster as additional noises and ignore the nesting structured of data. 


\section{Conclusion}

In a view of statistical modelling, this paper highlighted the use of variance components modelling approach as a promising gold standard in analysing DHS data given highlighted various nested level. We also propose for the future studies to consider more hierarchy levels associated with DHS data, although it may become more complicated extending for variance components approaches. To conclude, while high prevalence of modern contraceptive use is importantly recognised in fertility control, prevalence is still low in Tanzania with high fertility rate. This study emphases on the existences of village and street and region variabilities, the group level influence on modern contraceptive use that should be considered to ensure unequal allocation of family planning intervention between population levels.

\section{List of abbreviations:}

MCU Modern contraceptive use

ICC Intra-cluster correlations coefficient

DHS Demographic and health survey

WRA Women of reproductive age

PSU Primary sampling unit

\section{Acknowledgements}

Many thanks to African Institute of Mathematics (AIMS) Tanzania for funding support to attend conference presentation of this Manuscript. We thank Prof. A.C Mbago, Senior Lecturer at University of Dar es salaam, for inputs and encouragements toward preparation of this work. 
Again, we sincerely thank Mr. Tim Morries(UCL-UK), Paul Verdalind(Stockholm University) for many reviews of this work.

\section{Author's contribution}

All authors contributed to preparation of this work. Oliva Safari is the main author of this work. She designed the study, analyzed the data, presented the results, and prepared drafts for the manuscript. Dr Dunstan Bishanga (MD.PhD) provided reproductive health expertise and knowledge towards the preparation of this work. He commented on the public health impact of this research to the Tanzania settings. Dr Isambi Mbalawata(PhD) supported this work by many reviews from start to the end. He provided technical support on statistical modelling techniques to use of variance modeling approach.

Funding Not applicable

\section{Availability of data and material}

DHS data is publicity available at DHS website; www.dhsprogram.com.

\section{Ethics approval and consent to participate}

This study used secondary data, thus no ethical approval was required.

Consent for publication Not applicable

\section{Competing interests}

There is no conflict of interest to declare in preparation of this work. 


\section{References}

1. World Health Organisation (2006) Reproductive Health Indicators: Guidelines for their Generation, Interpretation and Analysis for Global Monitoring. WHO Publications, Geneva. https://doi.org/10.1016/S0277-9536(02)00341-6

2. Darmoul D, Baricault L, Sapin C, Chantret I, Trugnan G, Rousset M (1991) Health in 2015 from MDGs Millennium Development Goals to SDGs Sustainable Development Goals. World Health Organization,. https://doi.org/978 9241565110

3. United Nations, Department of Economic and Social Affairs PD (2015) Trends in Contraceptive Use Worldwide 2015. Contraception. https://doi.org/10.1016/j.contraception.2012.08.029

4. Smith R, Ashford L, Gribble J, Clifton D (2009) FAMILY PLANNING SAVES LIVES. Fourth Edition. Washington, DC USA

5. World Health Organisation (2010) WHO Progress Report. Reproductive health strategy to accelerate progress towards the attainment of intrenational developement goals and targets. Geneva, Switzerland

6. Singh S, Darroch E J (2012) Adding It Up: Costs and Benefits of Contraceptive Services Estimates for 2012. Guttmacher Institute and United Nations Population Fund (UNFPA), 201. https://doi.org/Available online at http://www.guttmacher.org/pubs/AIU-2012-estimates.pdf

7. The United Republic of Tanzania Ministry of Health and Social Wealfare (2013) The National Family Planning Costed Implementation Program. Dar es salaam

8. Price (2004) Addressing the Reproductive Health Needs and Right of Young People since ICPD- The Contribution of UNFPA and IPPF. Synthesis Report. University of Heidelberg

9. Ministry of Health, Community Development, Gender, Elderly and Children 
(MoHCDGEC) [Tanzania Mainland], Ministry of Health (MoH) [Zanzibar], National Bureau of Statistics (NBS), Office of the Chief Government Statistician (OCGS) and I

(2016) Tanzania Demographic and Health Survey and Malaria Indicator Survey (TDHSMIS) 2015-16. Dar es Salaam, Tanzania, and Rockville, Maryland, USA

10. Ferede T (2013) Multilevel Modelling of Modern Contraceptive Use among Rural and Urban Population of Ethiopia. American Journal of Mathematics and Statistics 3:1-16

11. Ejembi CL, Dahiru T, Aliyu AA (2015) Contraceptive Use in Nigeria. DHS Working Papers No. 120. Rockville, Maryland, USA: ICF International.

12. Kidayi PL, Msuya S, Todd J, Mtuya CC, Mtuy T, Mahande MJ (2015) Determinants of Modern Contraceptive Use among Women of Reproductive Age in Tanzania: Evidence from Tanzania Demographic and Health Survey Data. Advances in Sexual Medicine $05: 43-52$

13. Exavery A, Mubyazi GM, Rugemalila J, et al (2012) Acceptability of condom promotion and distribution among 10 - 19 year-old adolescents in Mpwapwa and Mbeya rural districts , Tanzania. BMC Public Health 12:1

14. Somba MJ, Mbonile M, Obure J, Mahande MJ (2014) Sexual behaviour, contraceptive knowledge and use among female undergraduates' students of Muhimbili and Dar es Salaam Universities , Tanzania : a cross-sectional study. 1-8

15. Kashagam E, Ngocho JS (2015) Prevalence Of Modern Contraceptive Methods Use Among Women Living With HIV Attending Care And Treatment Clinic At Amana Hospital Dar Es Salaam , Tanzania . 2:1740-1746

16. Mwangeni AE, Ankomah A, Richard AP (1998) Science : QUALITATIVE DATA ATTITUDES OF MEN TOWARDS FAMILY PLANNING IN MBEYA REGION, TANZANIA : A RURAL - URBAN COMPARISON OF QUALITATIVE DATA. Jouranl of Biosocial Scince 30:381-392 
17. Carle AC (2009) Methodology Fitting multilevel models in complex survey data with design weights : Recommendations. 13:1-13

18. ICF (2018) Demographic and Health Surveys Standard Recode Manual for DHS7. The Demographic and Health Surveys Program. Rockville, Maryland, U.S.A

19. Stephenson R, Baschieri A, Clements S, Hennink M, Madise N (2007) Contextual influences on modern contraceptive use in sub-Saharan Africa. American Journal of Public Health 97:1233-1240

20. Lasong J, Zhang Y, Gebremedhin SA, Opoku S, Abaidoo CS, Mkandawire T, Zhao K, Zhang H (2020) Determinants of modern contraceptive use among married women of reproductive age: A cross-sectional study in rural Zambia. BMJ Open 10:1-10

21. Sahai H, Ojeda MM (2005) Analysis of variance for random models. Analysis of Variance for Random Models. https://doi.org/10.1007/b138864

22. Merlo J (2002) Multilevel modelling of health statistics: Edited by A H Leyland, H Goldstein. New York: Wiley, 2001. Journal of Epidemiology \& Community Health. https://doi.org/10.1136/jech.56.7.560-a

23. Diez-roux A V (2000) Multilevel Analysis in Public Health Research. 21:171-92

24. Roux AVD (2002) A glossary for multilevel analysis. 588-595

25. Leeuw J De, Meijer E (2008) Introduction to multilevel analysis. Handbook of Multilevel Analysis. https://doi.org/10.1007/978-0-387-73186-5_1

26. Hox, Joop J., Moerbeek, Mirjam, van de Schoot R Multilevel Analysis: Techniques and Applications, Second Edition (Quantitative Methodology Series) 9781848728462: Amazon.com: Books. https://www.amazon.com/Multilevel-Analysis-ApplicationsQuantitative-Methodology/dp/1848728468. Accessed 10 Mar 2021

27. Makupe DJ, Kumwenda S, Kazembe L (2019) An application of mixed-effect models to analyse contraceptive use in Malawian women. Contraception and Reproductive 
Medicine 4:1-11

28. Wulifan JK, Brenner S, Jahn A, Allegri M De (2016) A scoping review on determinants of unmet need for family planning among women of reproductive age in low and middle income countries. BMC Women's Health. https://doi.org/10.1186/s12905-015-0281-3

29. Dias JG, Oliveira IT De (2015) Multilevel Effects of Wealth on Women's Contraceptive Use in Mozambique. 1-15

30. Mcguire C, Stephenson R (2015) Community Factors Influencing Birth Spacing among Married Women in Uganda and Zimbabwe. African Journal of Reproductive Health $19: 14-24$

31. Gary-webb TL, Baptiste-roberts K, Pham L, Wesche-thobaben J, Patricio J (2010) Neighborhood and weight-related health behaviors in the Look AHEAD ( Action for Health in Diabetes ) Study. https://doi.org/doi: 10.1186/1471-2458-10-312

32. Kruk ME, Rockers PC, Mbaruku G, Paczkowski MM, Galea S (2010) Community and health system factors associated with facility delivery in rural Tanzania : A multilevel analysis. Health policy 97:209-216

33. Belachew AB, Kahsay AB, Abebe YG (2016) Individual and community-level factors associated with introduction of prelacteal feeding in Ethiopia. Archives of Public Health $1-11$

34. Janevic T, Sarah PW, Leyla I, Elizabeth BH (2012) Individual and community level socioeconomic inequalities in contraceptive use in 10 Newly Independent States: a multilevel cross-sectional analysis. International Journal for Equity in Health 11:69

35. Vu LTH, Oh J, Bui QT-T, Le AT-K (2016) Use of modern contraceptives among married women in Vietnam: a multilevel analysis using the Multiple Indicator Cluster Survey (2011) and the Vietnam Population and Housing Census (2009). Global Health Action 9:29574 
36. Kaggwa E sther B, Diop N, Douglas J (2008) The Role of Individual and Community Normative Factors : A Multilevel Analysis of Contraceptive Use Among Women in Union in Mali. 34:79-88

37. Ngome E, Odimegwu C (2014) The social context of adolescent women's use of modern contraceptives in Zimbabwe: a multilevel analysis. Reproductive Health 11:64 
Figures
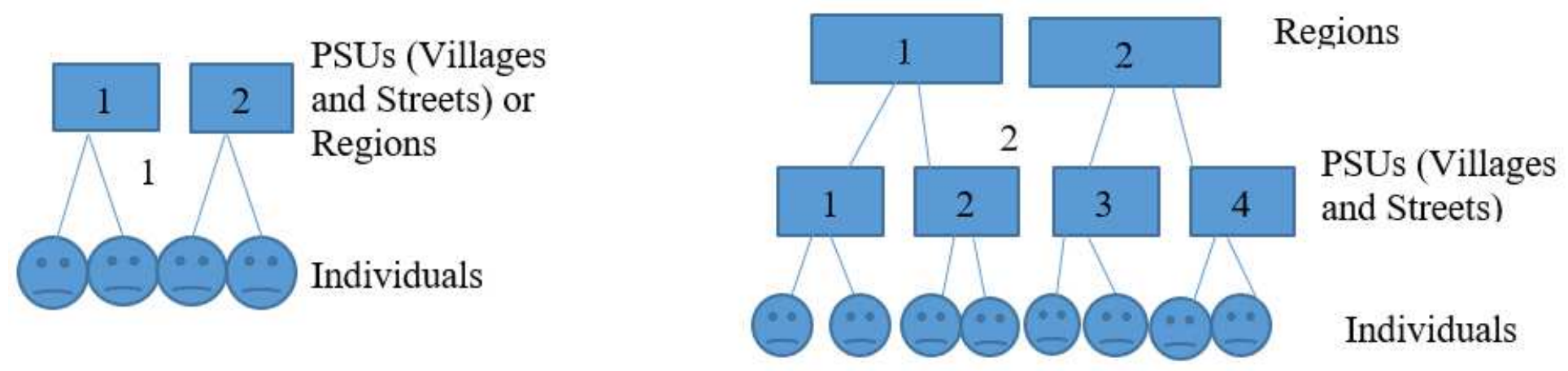

Figure 1

The TDHS data grouping structures two scenarios; (1). Individuals nested with in the villages and streets or individual nested with regions (two levels). (2); Individuals and villages and streets nested in regions (Three levels) 